\title{
BRAIN PORTION EXTRACTION USING HYBRID CONTOUR TECHNIQUE USING SETS FOR T1 WEIGHTED MRI OF HUMAN HEAD SCANS
}

\author{
K.Somasundaram and P.A.Kalaividya \\ Image Processing Lab, Department of Computer Science and Applications, \\ Gandhigram Rural Institute - Deemed University, Gandhigram-624302, \\ Tamil Nadu, India.
}

\begin{abstract}
Brain portion extraction from magnetic resonance image (MRI) of human head scan is an important process in medical image analysis. In this paper, we propose a computationally simple and a robust brain segmentation method. This method is based on forming a contour using the intensity values that satisfy a set property and detect the boundary of the brain. After detecting the brain boundary the brain portion is segmented. Experiments were conducted by applying the method on 3 volumes of T1 MRI data set collected from Internet Brain Segmentation Repository(IBSR) and compared the results with that of the popular skull stripping method Brain Extraction Tool (BET). The experimental results show that the proposed algorithm gives better results than that of BET.
\end{abstract}

\section{KEYWORDS}

Magnetic Resonance imaging(MRI), brain segmentation, contour tracing, connected component, set properties

\section{INTRODUCTION}

Magnetic resonance imaging (MRI) is an important diagnostic imaging technique to obtain high quality images of human organs for clinical and research purposes. It is a non-invasive, nonionizing and non-destructive imaging technique. It gives a high spatial resolution and an excellent contrast of soft tissues. To study brain related diseases like Alzheimer, dementia, tumor, brain injury, brain deformities etc MRI is used widely. MRI of a human head is captured in a series of 20-120 two dimensional (2D) slices. The collection of slices is called as a volume. These slices can be volume rendered to produce the 3D image of the 2D slices.MRI is taken in three orientations, axial (top to bottom), coronal(back to front) and sagittal (side to side) and in three types T1, T2 and PD weighted. MRI slices are to be analyzed by a neurologist/radiologist to know the pathology and structure of the brain. Further, there are several other image processing operations, like image registration, tumor detection, brain volume estimation, compression for telemedicine etc. Many of these operations are part of a frame work for computer assisted diagnostic(CAD) system. Therefore, it is essential to segment the brain portion from the MRI slices. This segmentation process is also known as skull stripping method, or brain extraction method..

Segmenting brain portion manually is a time consuming process and is operator biased. Therefore fully automatic methods are needed to segment brain portion from the MRI slices. 
Signal \& Image Processing : An International Journal (SIPIJ) Vol.7, No.1, February 2016

Semi-automatic segmentation methods also perform well for segmenting the brain.[1]-[3]. But the drawback of this method is, that they need some initial value or human intervention to start the algorithm.

To overcome the problems in semi-automatic methods fully automatic methods were developed in [4] - [8]. Jong and Lee.[4] proposed an algorithm, which needs histogram analysis to eliminate the background voxels. Brummer et al.[5] proposed a fully automatic algorithm that starts with a histogram-based thresholding preceded by an image intensity correction procedure. Lemieux et al.[6] proposed an automated algorithm to segment the brain portion from T1-weighted volume MRI. The algorithm uses automatic computation of intensity threshold and morphological operations. It is a three-dimensional method and therefore independent of scan orientation.

An automatic method for brain extraction was proposed by Stella and Blair [7]. This method uses an integrated approach which employs image processing techniques based on anisotropic filters, snake contouring technique and a priori knowledge to remove the eyes, and tricky structures in brain MRI. It is a multistage method. Segonne et al[8] proposed a hybrid segmentation method. Sadananthan etal[9] proposed a method based on graph cuts. Somasundaram and Kalaiselvi [10][11] have proposed methods based on intensity, morphological operation and largest connected component analysis. Somasundaram and Siva Shankar[12] proposed a brain extraction method using clustering and resonance principle. Somasundaram and Kalavathi [13] proposed a contour based brain extraction method. Somasundaram and Ezhilarasan[14] proposed a method based on gray scale transformation to detect the boundary of brain. A review of brain segmentation methods can be found in[15][16].

In this paper we propose a method to detect the brain boundary by forming a contour generated from a set of pixels that satisfy some intensity based boundary conditions in the slice. Using this contour we propose a scheme to extract brain portion from T1 weighted coronal MRI of human head scans. The remaining part of the paper is organized as follows. In section 2 , we give an outline of the basic principles used in our scheme. In section 3 we present our method. In section 4 results and discussions are given. In section 5 the conclusion is given. .

\section{BASIC PRINCIPLE USED IN SKULL STRIPPING}

In brain segmentation from a MRI slice, the main difficulty is to accurately detect the boundary separating the brain and non-brain tissues in the MRI slice. The proposed method traces a contour with property of a set, which satisfy a specified intensity values $(>180)$ in a grey scale MRI of human head scans. The set property and the governing equations are:

$\mathrm{A}=\{\mathrm{X}$ satisfies the property $\mathrm{P}\}$

$\mathrm{A}=\{(\mathrm{a}, \mathrm{b}) / \mathrm{b}-\mathrm{a}>180\}$

where, the property $\mathrm{P}$ of

$\mathrm{X}_{\mathrm{A}}=\{1: \mathrm{X}$ belongs to $\mathrm{A}, 0$ : $\mathrm{X}$ not belongs to $\mathrm{A}$

After finding the co-ordinates that satisfy the set property, contour of the brain is drawn to extract the brain. 


\section{Proposed Method}

The intensity features available in the T1 MRI slices are used to obtain brain boundary. In an MRI slice, the innermost region is brain. When we move from the center point of the brain towards the outer surface, first we come across brain-skull boundary(Figure 1). Most of the

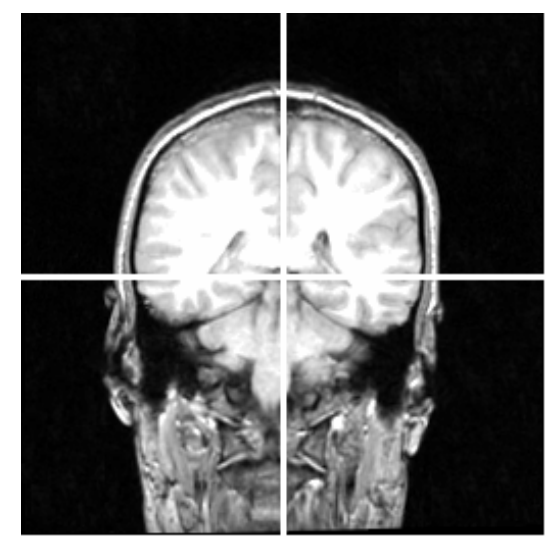

Figure 1. Sample T1 slice (Mid slice of the volume)

pixels representing CSF in a T1 slice is mostly $<180$. Very rarely pixels in CSF have intensity values more than 180 . Therefore, we may assume that the brain-skull boundary is demarcated by pixels with intensity $<180$ and others with intensity $>=180$ (brain). We use this property and assign the pixels values to either of $a$ or $b$ in the set $A$ as given in eqns.(1)-(3).

When we move from the centre of the slice, intensity at each co-ordinate points will come under the property of the Set defined in equation (3). The points which have the intensity values satisfying the property of the Set A will be labeled as in the region otherwise it is not labeled. Once the first Set property is achieved, we move back in the same row and perform the set property analysis. We repeat this for all rows of the image by starting mid point in each row.When the regions are labelled, it is possible to find the distinct region. Usually, the mid slice in a MRI volume will contain brain as the largest connected area. But the slices in lower and upper slices will contain brain in more than one connected region. For such cases, the largest connected component (LCC), out of many regions, in the given image is traced out. The run length identification scheme for region labelling described by Sonka et al. [17] is used to find the LCC among the region as:

$\mathrm{R}_{\mathrm{LCC}}=\mathrm{R}\left(\arg \max \mathrm{R}_{\mathrm{A}}(\mathrm{i})\right)$

where, $R_{A}(i)$ is the area of $i^{\text {th }}$ region $R(i)$.

The process chart of the proposed method is shown in Figure 2. 
Signal \& Image Processing : An International Journal (SIPIJ) Vol.7, No.1, February 2016

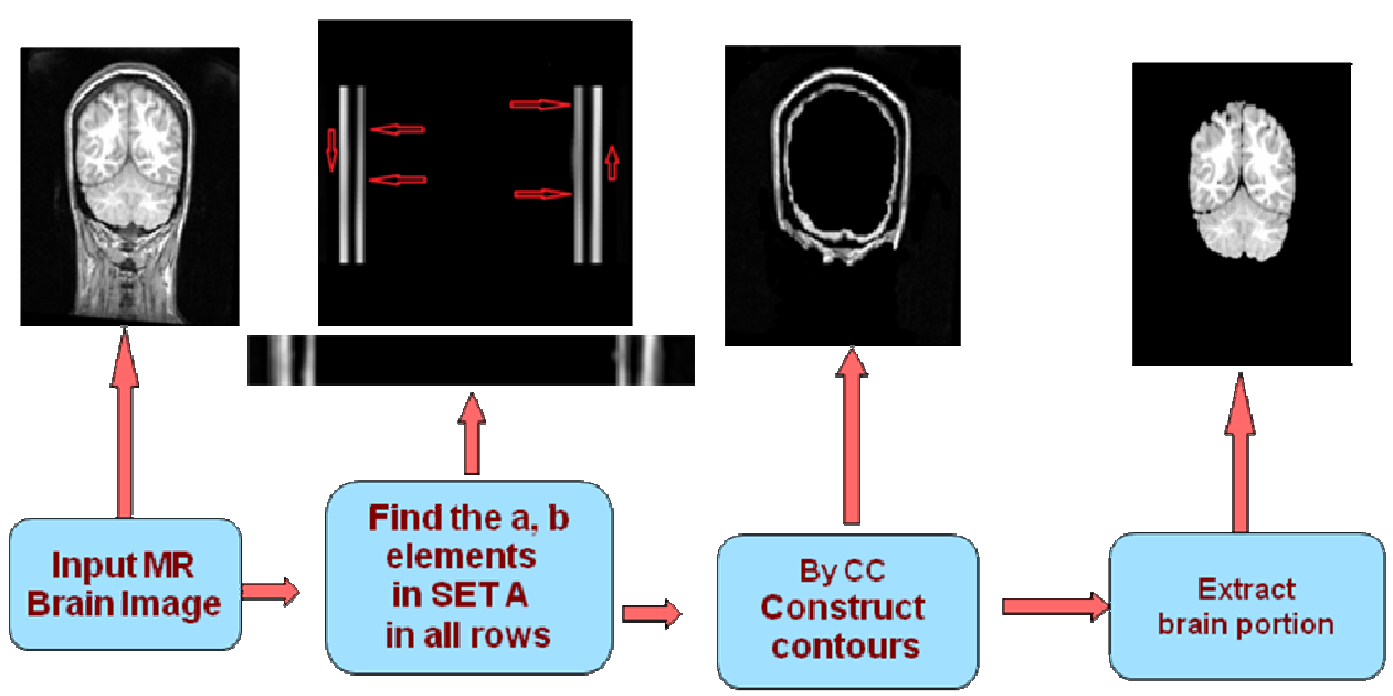

Figure 2. Process chart of the proposed method

In MRI head scans, the middle slice of the volume contains brain as a single largest region. Identification of brain portion in the middle slice is easy and the brain region in this is used as initial reference to proceed throughout the volume. We start the process at the middle slice of the MRI volume at slice number= N/2 approximately, where $\mathrm{N}$ is the total number of slices in the volume. Then the brain mask is obtained from the middle slice and used as a reference to extract brain from adjacent slices lying above and below it. This process will propagate from the middle slice and move to lower slices and then from middle slice to upper slices, one direction at a time and produce the brain mask of each slice in the volume. Using the brain mask, the brain portion is extracted from the input slice .

\subsection{Algorithm}

Step 1. Read middle slice

Step 2. Find midpoint of the mid slice in the MR Image.

Step 3: Apply the SET property to define the regions having intensity values more than 180 adn assign to the Set.

Step 4: Draw the contour of the pixels satisfying the Set property.

Step 5: Is there more than one connected region?

IF yes, then perform LCC (using eqn.(4)) and

take LCC as brain mask(B)

Else take the contour as brain mask (B)

Step 6: Segment brain portion using B

Step 7: Take B as reference mask for adjacent slices and repeat step 2 to step 6 for all slices from middle slice to top slices and middle slice to bottom slices.

\subsection{Materials Used}

We have used three volumes of MRI T1 coronal datasets taken from IBSR website developed by Centre for Morphometric Analysis (CMA) at Massachusetts General Hospital, for the proposed 
method [18]. The Slice thickness is $3.0 \mathrm{~mm}$ and each of 256X256 in size. The hand segmented result, gold standard is also available in the IBSR .

\subsection{Performance Evaluation Metrics}

To evaluate the performance of the proposed method we make use of similarity indices Jaccard [19] and Dice coefficient[20]. The Jaccard and Dice coefficients are the parameters giving the amount of overlap between two data sets $\mathrm{A}$ and $\mathrm{B}$. The value of $\mathrm{J}$ and $\mathrm{D}$ varies from 0 for total disagreement and 1 for complete agreement, between $\mathrm{A}$ and $\mathrm{B}$.

$$
\begin{aligned}
& J(A, B)=\frac{|A \cap \bar{B}|}{|A \cup B|} \\
& D(A, B)=\frac{2|A \cap B|}{|A|+|B|}
\end{aligned}
$$

\section{RESULTS AND DISCUSSION}

We carried out experiments by applying the proposed hybrid contour method on three volumes of MRI T1 coronal datasets of human head scans and made quantitative and qualitative performance analysis of the method by computing the similarity coefficients $J$ and $D$ between the extracted brain portions and the hand segmented Gold standard available in IBSR

The computed values of $\mathrm{J}$ and $\mathrm{D}$ by the proposed method and that of the popular method, Brain Extraction Tool (BET) [21] are given in Table 1 and are plotted in Figure 3. We observe from Table 1 and Figure 3, that the proposed method gives better values for $\mathrm{J}$ and $\mathrm{D}$ than that of BET for the three volumes.

Table 1. Computed average values of Jaccard similarity index and Dice Co-efficient.

\begin{tabular}{|c|c|c|c|c|}
\hline \multirow{2}{*}{$\begin{array}{l}\text { Data set } \\
\text { T1 }\end{array}$} & \multicolumn{2}{|l|}{ BET } & \multicolumn{2}{c|}{ Proposed method } \\
\cline { 2 - 5 } & J & D & J & D \\
\hline $1 \_24$ & .9459 & .9722 & $\mathbf{. 9 5 6 8}$ & .9746 \\
\hline $13 \_3$ & .9453 & .9615 & $\mathbf{. 9 5 9 5}$ & .9786 \\
\hline $4 \_8$ & .9531 & .9760 & .9583 & $\mathbf{. 9 8 6 7}$ \\
\hline
\end{tabular}

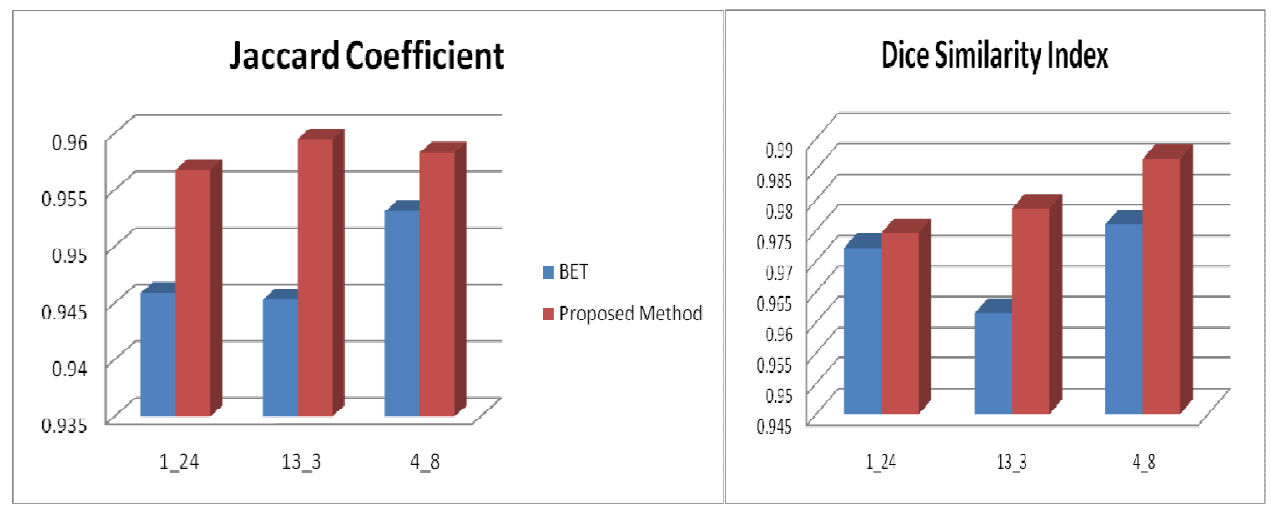

Figure 3 Computed values of $\mathrm{J}$ and $\mathrm{D}$ for the proposed and BET methods 
Signal \& Image Processing : An International Journal (SIPIJ) Vol.7, No.1, February 2016

For qualitative performance, the segmented brain using our method for one volume of MRI are shown in Fig.4

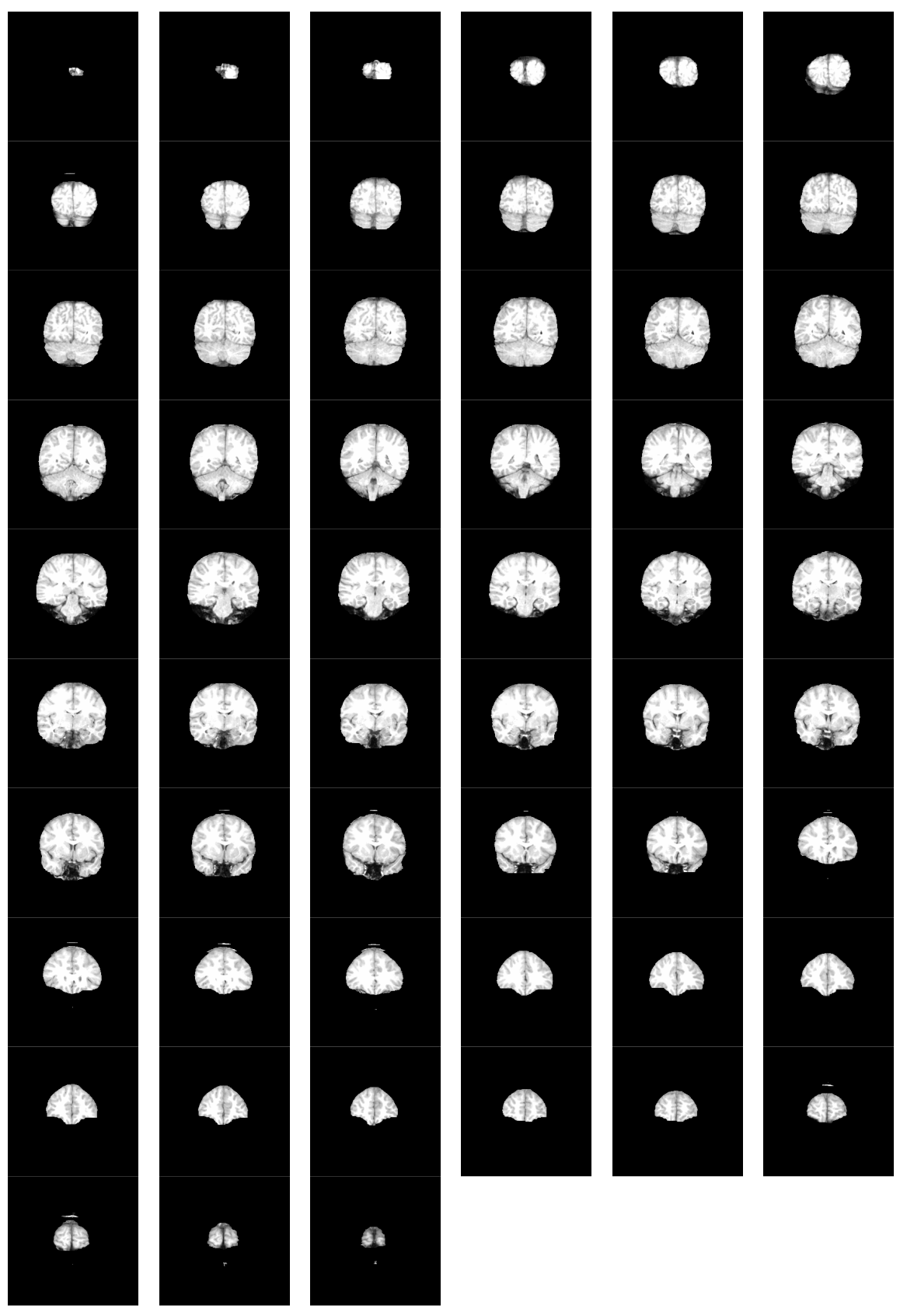

Figure 4. Extracted brain portions from T1 weighted coronal 13_3 using our method

To qualitatively compare our results with that of BET method, brain portions segmented by the proposed method, hand segmented result and by BET are shown in Figure 5.The second column shows the original slices, third column the Gold standard, fourth column by the proposed method and fifth column by BET. 
Signal \& Image Processing : An International Journal (SIPIJ) Vol.7, No.1, February 2016

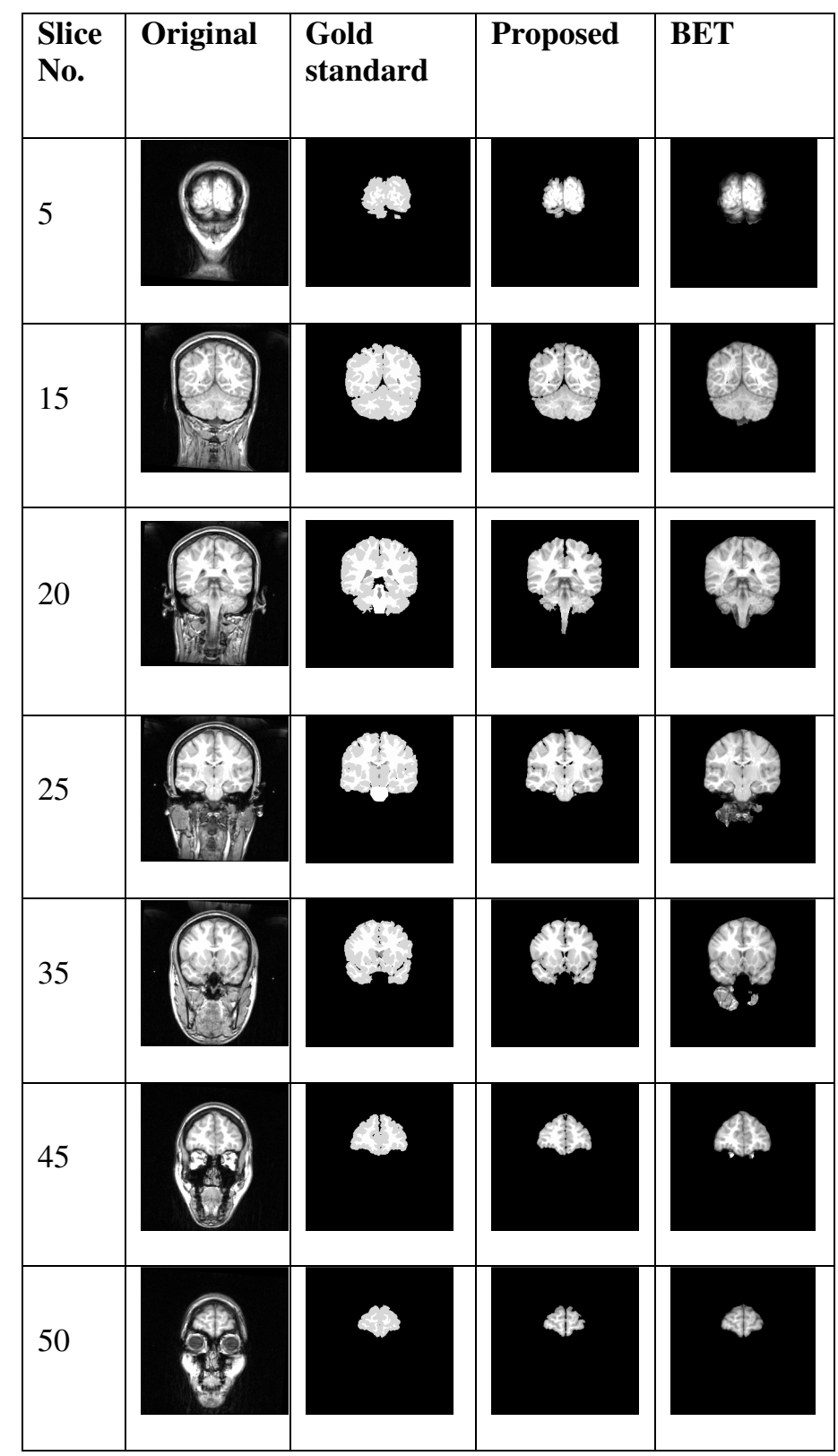

Figure 5. Segmented brain portions. Second column shows the original slices, second shows the Gold standard, third by the proposed method and fifth by BET.

We not from row 3 and 4 of Figure 5, that the proposed method performed well where BET failed.

\section{CONCLUSIONS}

In this paper, we have proposed contour tracing technique by forming a set that specifies the boundary pixels. Using the contour the brain boundary is detected. Using this techniques we have extracted the brain portions from T1 weighted coronal MRI of human head scans. Experimental results on three volumes of MRI shows that the proposed method gives better results than that of the popular BET method in terms of Jaccard index and Dice coefficients. The proposed method 
Signal \& Image Processing : An International Journal (SIPIJ) Vol.7, No.1, February 2016

gives the best value of 0.9595 for $\mathrm{J}$ and 0.987 for $\mathrm{D}$. The proposed method is computationally simple.

\section{REFERENCES}

[1] Adams, R. and Bischof, L., Seeded region growing, IEEE Trans. Pattern Anal. Mach. Intell. vol 16, pp.641-646., 1994.

[2] Hohne.K.H and Hanson.W.A., Interactive 3D segmentation of MRI and CT volumes using morphological operations. J. of Comput.Assist. Tomogr. vol.16, pp. 285-294, 1992

[3] Justice, R.K., Stokely, E.M., Strobel, J.S., Ideker, R.E. and Smith, W. M., Medical image segmentation using 3D seeded region growing. Proc. SPIE Med. Imag. vol.3034 ,pp.900-910, 1997.

[4] Jong Geun park, Chulhee Lee, Skull Stripping based on Region growing for Magnetic resonance brain images, NeuroImage, vol.40, pp.1394-1407, 2009

[5] Brummer, M.E., Mersereau, R.M., Eisner, R.L., and Lewine, R.R.J.. Automatic detection of brain contours in MRI data sets. IEEE Trans. Med. Imag. vol.12, pp.153-166, 1993.

[6] Lemieux, L., Hagmann, G., Krakow, K., and Woermann, F.G. Fast, accurate, and reproducible automatic segmentation of the brain T1-Weighted volume MRI data. Mgn. Reson. Med. vol.42, pp. 127-135., 1999.

[7] Stella Atkins, and Blair T Mackiewich, Fully Automatic segmentation of the Brain in MRI, IEEE transactions on Medical Imaging, vol.17, pp.98-107, 1998.

[8] Segonne F, Dale AM, Busa E, Glessner M, Salat D, Hahn HK, and Fischl B, A hybrid approach to the skull stripping problem in MRI., Neuroimage, vol.22, pp.1060-1075, 2004.

[9] Sadananthan SA, Zheng W, Chee MW, and Zagorodnov V., Skull stripping using graph cuts, Neuroimage, vol.49. pp. 225-239, 2010.

[10] Somasundaram K and Kalaiselvi T, Fully automatic brain extraction algorithm for axial T2-weighted magnetic resonance images, Computers in Biology and Medicine, vol.40, pp. 811-822, 2010.

[11] Somasundaram $\mathrm{K}$ and Kalaiselvi $\mathrm{T}$, Automatic brain extraction methods for $\mathrm{T} 1$ magnetic resonance images using region labeling and morphological operations, Computers in Biology and Medicine, vol. 41, pp.716-725, 2011.

[12] Somasundaram.K. and Siva Shankar.R, Skull Stripping of MRI Using Clustering and Resonance method, International Journal on Knowledge Management \& E-Learning, vol.3, pp.19-23,2011.

[13] Somasundaram K and Kalavathi P, Contour-Based Brain Segmentation Method for Magnetic Resonance Imaging Human Head Scans, Journal of Computer Assisted Tomography, vol. 37, pp 353368, 2013.

[14] Somasundaram K ans Ezhilarasan K, Automatic Brain Portion Segmentation from Magnetic Resonance Images of Head Scans using Gray Scale Transformation and Morphological Operations, Journal of computer assisted tomography, vol. 39, pp. 552-558, 2015.

[15] Balafar,M.A., Ramli, A.R.,Saripan, M.I, and Mashohor, S., Review of brain MRI image segmentation, Artificial Intelligence Review, vol.33, pp. 262-274,2010

[16] Kalavathi, P. and Surya Prasath, V.B. Methods on Skull Stripping of MRI Head scan images- A Review, Journal of Digital Imaging, First online, pp.1-15,Dec. 2015 
Signal \& Image Processing : An International Journal (SIPIJ) Vol.7, No.1, February 2016

[17] Milan Sonka, Vaclav Hlavac and Roger Boyle, Image Processing, Analysis and Machine Vision, Thomson learning Inc, second edition, 2007.

[18] International Brain Segmentation Repository, Center for Morphometric Analysis Massachusetts General Hospital, CNY-6, Building 149, 13th Street, Charlestown, MA, 02129-USA. http://www.cma.mgh.harvard.edu/ibsr/ibsr_data/sec3.sub2.html

[19] Jaccard P., The Distribution of Flora in Alpine Zone, New Phytol, vol.11, pp.37-50,1912

[20] Dice L., Measures of the Amount of Ecologic Association between Species, Ecology, vol.26, pp.297302, 1945.

[21] Smith S.M .,Fast robust automated brain extraction, Human Brain Mapping, vol.17, pp.143-155, 2002.

\section{AUTHORS}

Somasundaram K, received his Master of Science (M. Sc.) degree in Physics from the University of Madras, Chennai, India in 1976, the Post Graduate Diploma in Computer Methods from Madurai Kamaraj University, Madurai, India in 1989 and the $\mathrm{Ph}$. D. degree in theoretical Physics from Indian Institute of Science, Bangalore, India in 1984. He is presently working as Professor at the Department of Computer Science and Applications, Gandhigram Rural Institute, Dindigul, India. From 1976 to 1989, he was a Professor with the Department of Physics at the same Institute. He was senior Research Fellow of council of Scientific and

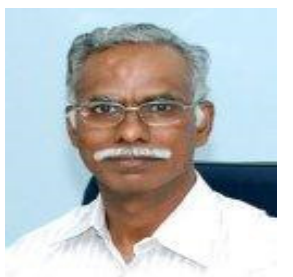
industrial Research(CSIR),Govt. of India in 1983. He was previously a Researcher at the International Centre for Theoretical Physics, Trieste, Italy and Development Fellow of Commonwealth Universities, at Edith Cowan University, Perth, Australia. His research interests are image processing, image compression and medical image processing. He is Life member of Indian Society for Technical Education, India and Life member in Telemedicine society of India. He is also an annual member of IEEE USA

Kalaividya P A, received her Bachelor of Physics (B.Sc.) degree in 2006 and the Master of Computer Applications (M.C.A.) degree in 2009 from the Gandhigram Rural Institute-Deemed University(GRI), Dindigul, TN, India. She is currently pursuing the Ph.D. degree with the Department of Computer Science and applications, GRI. Her research interests is image processing

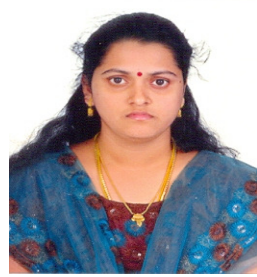

